\title{
Empirical Mode Decomposition of the acceleration response of a prismatic beam subject to a moving load to identify multiple damage locations
}

\author{
J. Meredith, A. González* and D. Hester \\ Civil Engineering School, University College Dublin, Newstead, Belfield, Dublin, Ireland
}

\begin{abstract}
Empirical Mode Decomposition (EMD) is a technique that converts the measured signal into a number of basic functions known as intrinsic mode functions. The EMD-based damage detection algorithm relies on the principle that a sudden loss of stiffness in a structural member will cause a discontinuity in the measured response that can be detected through a distinctive spike in the filtered intrinsic mode function. Recent studies have shown that applying EMD to the acceleration response, due to the crossing of a constant load over a beam finite element model, can be used to detect a single damaged location. In this paper, the technique is further tested using the response of a discretized finite element beam with multiple damaged sections modeled as localized losses of stiffness. The ability of the algorithm to detect more than one damaged section is analysed for a variety of scenarios including a range of bridge lengths, speeds of the moving load and noise levels. The use of a moving average filter on the acceleration response, prior to applying EMD, is shown to improve the sensitivity to damage. The influence of the number of measurement points and their distance to the damaged sections on the accuracy of the predicted damage is also discussed.
\end{abstract}

Keywords: Empirical Mode Decomposition, vibration-based analysis, damage detection, signal decomposition

\section{Introduction}

The process of determining and tracking structural integrity and assessing the nature of damage in a structure is often referred to as health monitoring. In health monitoring, it is advantageous, especially in the case of bridges, to use the structural response from operational dynamic loads, such as vehicular loads, for damage detection. Vibration-based structural damage detection methods that use this response to locate damage, are based on the fact that damage in a structure usually implies a local stiffness reduction or increase in flexibility, which induce changes in its dynamic properties [1]. Time series analysis is receiving increased attention in the field as it permits vibration signals, from structures, to be decomposed into fundamental basis functions that are used to characterise the vibration response. In the past, extracting damage location and magnitude using vibration-based methods without undamaged data to compare to has been a major problem for researchers. According to Doebling [2], techniques that are to be seriously considered for implementation in the field should demonstrate that they can perform well under the limitations of a small number of measurement locations, and under the constraint that these locations be selected a priori without knowledge of the damage location. Wavelet analysis and Empirical Mode Decomposition (EMD), as part of the Hilbert-Huang Transform, are two widely researched time-series analysis methods with the potential to meet these requirements. In contrast to the Fourier transform, which maps from the time domain to the frequency

*Corresponding author. E-mail: arturo.gonzalez@ucd.ie. 
domain, wavelet analysis and EMD may be applied and mapped to the space or time domain of the structure therefore making them capable of damage location.

\subsection{Wavelet analysis}

Wavelet analysis, in recent years, has been one of the emerging and fast-evolving mathematical and signal processing tools for vibration-based analysis. It has been used to locate discontinuities in dynamic measurements that could be associated with structural damage. Kim and Melham [3] describe wavelet analysis as a breaking up of a signal into shifted and scaled versions of a mother wavelet or basis function. This results in variable sizes of a window function and makes it possible to detect discontinuities and breakdown points of data. Wavelet transforms may be applied and mapped to the space or time domain of the structure therefore making it capable of damage location. Zhu and Law [4] first suggested that by performing a continuous wavelet transform (CWT) on the deflection-time signal of a cracked beam subject to a constant moving load, it was possible to determine the position of the crack. Ayenu-Prah and Attoh-Okine [5] however highlight that there are complications that arise out of defining a priori basis functions, these being that a wavelet based interpretation of a signal is only meaningful relative to the selected mother wavelet. Other disadvantages they mention include spectral leakage and the fact it is non-adaptive. The disadvantages of Wavelet analysis prompted the development of a new signal processing method, EMD. According to Huang et al. [6], the EMD method can deal with data from nonstationary and nonlinear processes, and in contrast to other methods has an a posterior defined basis.

\subsection{Empirical Mode Decomposition}

EMD is a signal processing method which decomposes data into several intrinsic mode functions (IMFs) by a procedure known as the sifting process. An IMF must satisfy two conditions: (1) Within the data range, the number of extrema and the number of zero crossings are equal or differ by one only; and (2) the envelope defined by the local maxima and the envelope defined by the local minima are symmetric with respect to the mean. Huang and Shen [7] provide a detailed description of how EMD is performed, the following being a brief outline.

The sifting process is executed by first constructing an upper and lower envelope of the signal, by connecting its local maxima and minima, using a cubic spline (Fig. 1a). The mean of the two envelopes is then found and subtracted from the original signal, resulting in a signal called the first IMF. The difference between the first IMF and the original signal is then subjected to the same sifting process, giving the second IMF. The sifting process continues

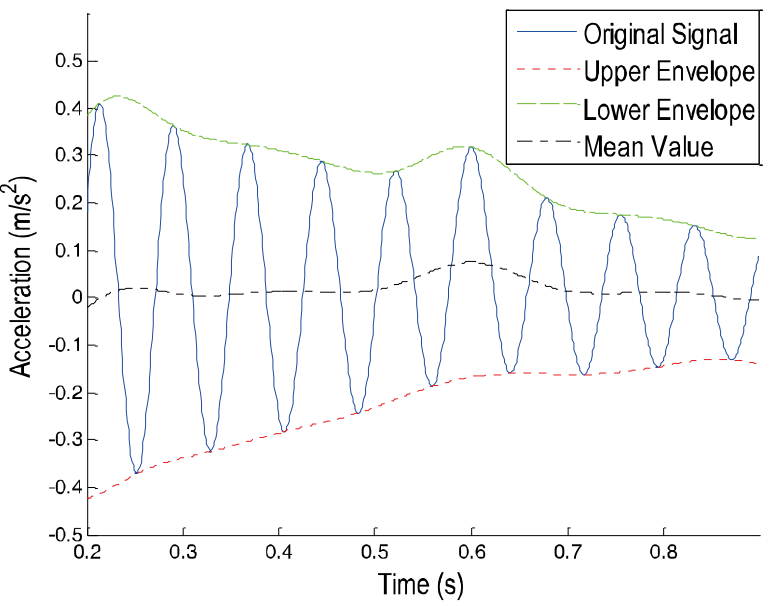

(a)

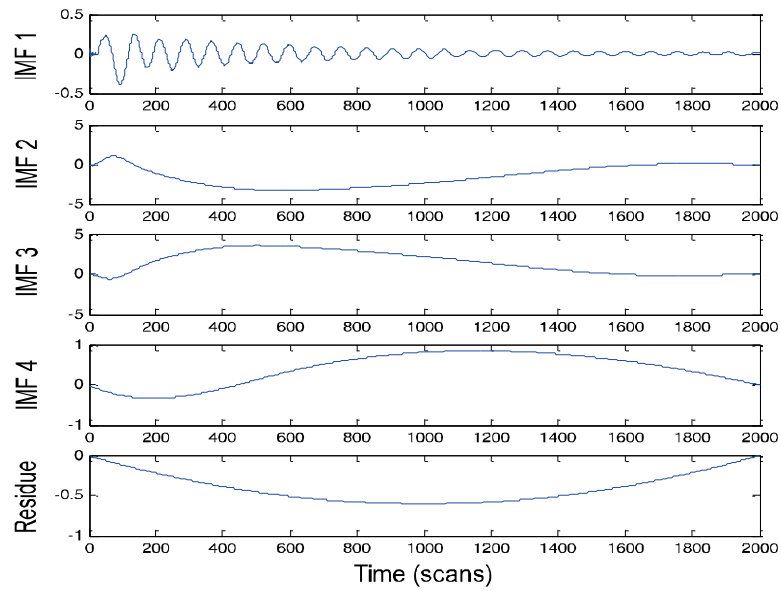

(b)

Fig. 1. (a) Construction of upper and lower signal envelopes and their mean value, (b) IMF plots of acceleration signal. 


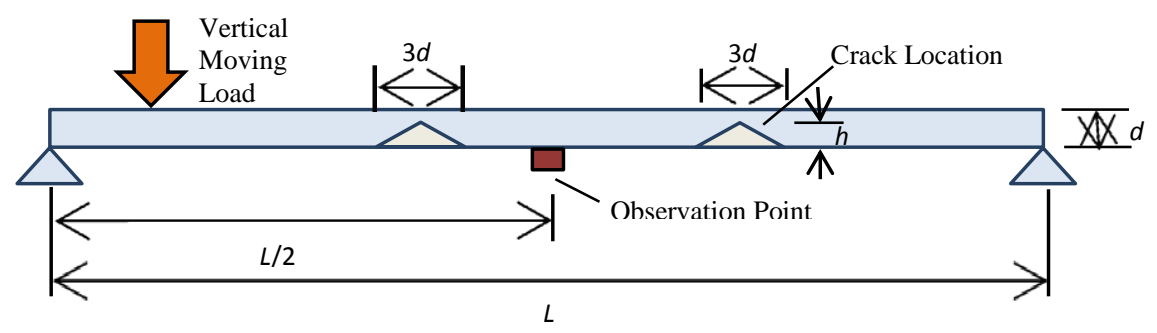

Fig. 2. Damaged beam model.

until the residue signal reaches a predetermined value or becomes a monotonic function. The first IMF contains the highest frequency components in the signal while the residue represents the lowest frequency components (Fig. 1b).

To detect discontinuities related to damage in a structural response, an intermittency frequency, $f_{c}$, has been traditionally applied to an IMF so that the data having frequencies lower than $f_{c}$ will be removed from the filtered IMF. According to Yang et al. [8], $f_{c}$ should be smaller than the frequency of discontinuity but larger than the highest structural frequency appearing in the measurement. The EMD-based damage detection algorithm relies on the principle that a sudden loss of stiffness in a structural member will cause a discontinuity in the measured response that can be detected through a distinctive spike in the filtered IMF. The IMFs with higher frequency components are more sensitive to this discontinuity.

EMD has been recently applied to damage identification in structures subject to dynamic loading. Xu and Chen [9] conducted experiments on the use of EMD using a three storey steel frame building model. A sudden change of structural stiffness was simulated and signals were acquired using accelerometers. The measured structural response time history from each test case was processed using the EMD approach with intermittency check. The first IMF components were then used to identify the damage time instant and damage location in the building. $\mathrm{Xu}$ and Chen concluded that the EMD approach can accurately identify the damage time instant by observing the occurrence time of the damage spike in the first IMF component of the acceleration response. They found it to be a useful tool for damage detection of real structures in the sense that it is a signal based and model free method requiring no prior knowledge of the structure.

Yang et al. [8], Pines and Salvino [10] and Yinfeng et al. [11] also demonstrated the ability of EMD to detect structural damage, however, it had not been tested for structures subject to moving loads until Bradley et al. [12]. This investigation concluded that EMD could be used to detect damage from the accelerations of a beam model subject to the crossing of a load. They applied a high-pass filter to the IMFs, resulting from the acceleration response, to detect a single damaged location. It was found that high levels of noise and long beam lengths introduced some small inaccuracies; however, these could be reduced with an increase in observation points. Roveri and Carcaterra [13] used EMD before applying the Hilbert transform, a method known as the Hilbert-Huang transform, on signals obtained from a beam modelled with an open crack and subjected to a moving load to obtain the instantaneous frequency. They found that when the load crossed the cracked section a sharp peak occurred however, results were sensitive to ambient noise and only crack depths of $25 \%$, or greater, of the section height were detected accurately. Overall, EMD has been shown to be an adaptive analysis method with no a priori knowledge required.

In this paper, this EMD technique is further tested using simulations of the response of a beam with two damaged locations. Results reveal that there are no significant differences between filtering the IMF or filtering the original signal. The authors suggest an alternative approach that improves the sensitivity of the algorithm to damage.

\section{Model set-up}

EMD's capabilities are examined using a finite element Euler-Bernoulli beam, modelled in Matlab 7 (Fig. 2). The program is set up to calculate the dynamic response of the simply supported beam, with damaged elements, subject to a constant force traversing it. The beam is made up of a number of discretized elements each with two nodes. Each node has two degrees of freedom, rotation and vertical displacement. 
The program first defines the elemental stiffness $(K e)$ and mass $(M e)$ beam matrices. The stiffness matrix is calculated using the force method and the mass matrix is calculated using the consistent mass matrix formulation (Tedesco [13]), with the resulting elemental matrices given by:

$$
\begin{aligned}
& K e=\left[\begin{array}{cccc}
\frac{12 E I}{L_{e}^{3}} & \frac{6 E I}{L_{e}{ }^{2}} & -\frac{12 E I}{L_{e}{ }^{3}} & \frac{6 E I}{L_{e}{ }^{2}} \\
\frac{6 E I}{L_{e}{ }^{2}} & \frac{4 E I}{L_{e}} & -\frac{6 E I}{L_{e}{ }^{3}} & \frac{2 E I}{L_{e}} \\
-\frac{12 E I}{L_{e}^{3}} & -\frac{6 E I}{L_{e}{ }^{2}} & \frac{12 E I}{L_{e}{ }^{3}} & -\frac{6 E I}{L_{e}{ }^{2}} \\
\frac{6 E I}{L_{e}{ }^{2}} & \frac{2 E I}{L_{e}} & -\frac{6 E I}{L_{e}{ }^{2}} & \frac{4 E I}{L_{e}}
\end{array}\right] \\
& e=\frac{\rho A L_{e}}{420}\left[\begin{array}{cccc}
156 & 22 L_{e} & 54 & -13 L_{e} \\
22 L_{e} & 4 L_{e}^{2} & 13 L_{e} & -3 L_{e}^{2} \\
54 & 13 L_{e} & 156 & -22 L_{e} \\
-13 L_{e} & -3 L_{e}^{2} & -22 L_{e} & 4 L_{e}^{2}
\end{array}\right]
\end{aligned}
$$

where $E$ is the modulus of elasticity, $I$ is the moment of inertia, $A$ is the cross-sectional area, $\rho$ is the density and $L_{e}$ is the length of the elemental beam. The elemental matrices, in Eqs (1) and (2), are for undamaged elements. A crack is modelled as a reduction in stiffness of the beam in the area of the crack with the assumption that the crack does not affect the mass of the structure. The severity or depth of the crack is described by the variable $\delta$ which is the ratio of the crack height $(h)$ to the beam depth $(d)$. The area of influence of this stiffness reduction is approximated with a triangular shape which extends $1.5 \mathrm{~d}$ either side of the crack, in accordance with Sinha et al. [15]. New EI values are calculated for the damaged elements and the associated elemental matrices in the crack region are modified to allow for this. The global stiffness $(K g)$ and mass $(M g)$ matrices are then assembled and the boundary conditions are applied. Next the forcing vector is created with relation to the time of the vehicle to cross the bridge and distributed to the nodes of the underlying beam element using shape functions. The equation of motion for a general undamped multi-degree of freedom system, with $n$ degrees of freedom, subject to an external force is given by:

$$
[M g]\{\ddot{y}\}+[K g]\{y\}=\{F\}
$$

Where $[K g]$ and $[M g]$ are the symmetric $n \times n$ stiffness and mass matrices respectively, $\{y\}$ and $\{\ddot{y}\}$ are the $n \times 1$ displacement and acceleration vectors respectively, and $\{F\}$ is the $\mathrm{n} x 1$ vector of external forces. The equation of motion is integrated using the Wilson Theta method (theta $=1.4$ ) and the displacement, velocity and acceleration vectors are obtained. The mode superposition method is generally the most common method applied, however, according to Tedesco [13], it is not suitable for multi-degree of freedom systems with nonlinearities, such as cracks, these systems requiring direct integration to evaluate the dynamic response.

For testing purposes, the beam is assumed to be made of material with a modulus of elasticity $E$ of $34 \mathrm{GPa}$ and a density $\rho$ of $2400 \mathrm{~kg} / \mathrm{m}^{3}$. The beam cross-section is uniform of width $0.5 \mathrm{~m}$ and depth $1 \mathrm{~m}$. Unless otherwise specified, acceleration signals are simulated at the mid-span of the beam, at a scanning rate of $1000 \mathrm{~Hz}$, as a $100 \mathrm{kN}$ load travels across the structure. 


\section{Damage detection based on Empirical Mode Decomposition}

\subsection{Original approach using highpass filter}

As reviewed in Section 1.2, the traditional use of this approach typically consists of applying EMD to the acceleration signal followed by a high-pass filter, set to the required $f_{c}$. To demonstrate the capabilities of this method in detecting multiple damage locations an example is demonstrated here where the load is moving at a velocity of $30 \mathrm{~m} / \mathrm{s}$ over a $10 \mathrm{~m}$ beam span. Cracks of $\delta=0.3$ are located at $4 \mathrm{~m}$ and $8 \mathrm{~m}$ from the first support. Figures $3 \mathrm{a}$ and $3 \mathrm{~b}$ show the first IMF of the acceleration signal before and after filtering. Damage spikes can be seen around 0.133 and $0.266 \mathrm{~s}$ which correspond to $4 \mathrm{~m}$ and $8 \mathrm{~m}$ respectively on the beam model. The initial occurrence of damage spikes starts approximately $0.05 \mathrm{~s}$ previous to the location of the crack and tapers off to approximately $0.05 \mathrm{~s}$ after, which is equivalent to the triangular shape stiffness reduction of $1.5 \mathrm{~d}$ employed to either side of the crack (i.e., $1.5 \mathrm{~m}$ is covered in $0.05 \mathrm{~s}$ at $30 \mathrm{~m} / \mathrm{s}$ ). Figures $3 \mathrm{c}$ and $3 \mathrm{~d}$ show the original acceleration signal and the signal after applying a high-pass filter.
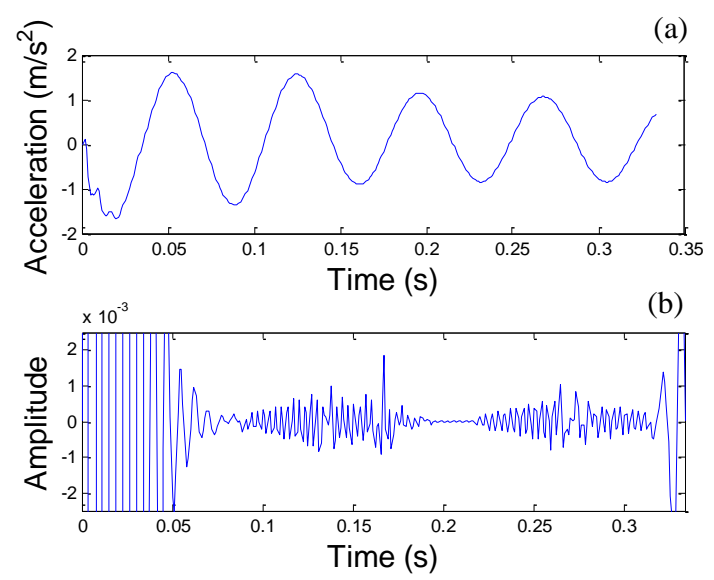
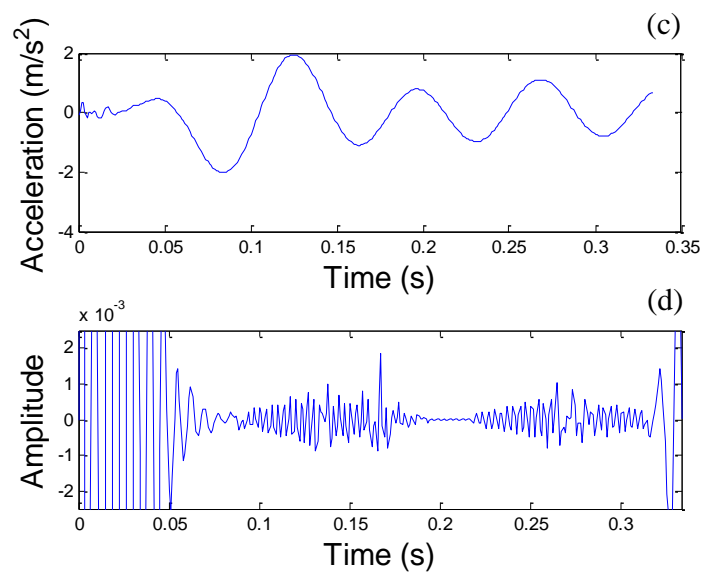

Fig. 3. (a) IMF 1 and (b) filtered IMF 1, (c) acceleration signal and (d) filtered acceleration signal.

Figures $3 \mathrm{~b}$ and $3 \mathrm{~d}$ show a large transient occurring at the beginning of the filtered signal. The reasons for this transient are twofold. Firstly, it can be expected as a result of the filtering process. The filter encounters a change in frequency at the start up of the signal, which is greater than $f_{c}$, leading to large initial spiking. Secondly, the original acceleration signal has a start-up discontinuity as the load moves onto the bridge. Spiking also occurs at the sensor location (i.e. $0.166 \mathrm{~s}$ ). These discontinuities are of a high frequency which pass through the filter and also contribute to the transient. It can be seen that both Figs $3 \mathrm{~b}$ and $3 \mathrm{~d}$ show results that are almost identical. Further testing showed that the IMF signal does not appear to provide any more prominent detail compared to the directly filtered acceleration signal and it was therefore concluded that EMD applied in this manner has no significant advantage over using the original signal for detecting the discontinuity associated to damage. Also it was noted that the selection of $f_{c}$ when applying the filter was based on a subjectively selected scale and therefore the method was no longer adaptive. An alternative approach is therefore suggested which overcomes the drawbacks of this approach.

\subsection{Alternative approach using moving average filter}

A new approach that reduces the effect of the discontinuity at the start of the filtered signals, is adaptive and improves the sensitivity of the processed signal to the presence of damage is introduced here. This approach is based on the use of a moving average filter (MAF) prior to applying EMD, rather than the high-pass filter applied afterwards as demonstrated in the previous section. The total acceleration is shown in Figs $3 \mathrm{c}$ and $4 \mathrm{a}$ for damaged and healthy beams respectively. Total acceleration can be assumed to consist of a static component and a dynamic 


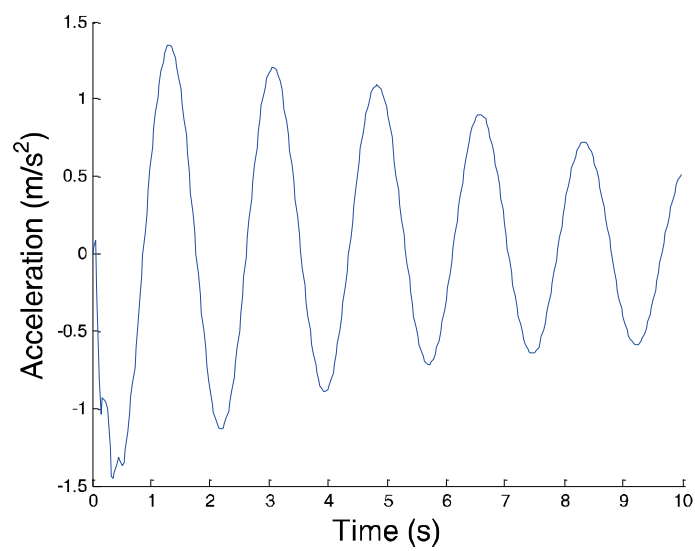

(a)

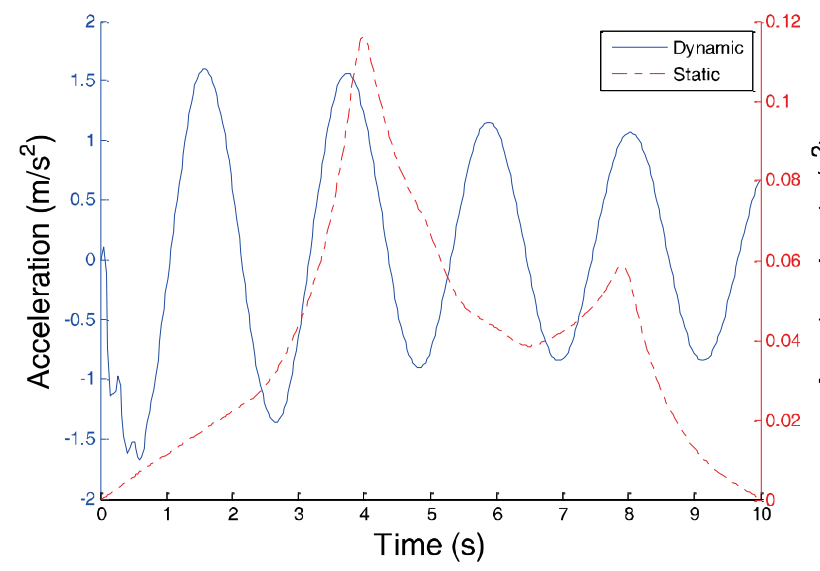

(c)

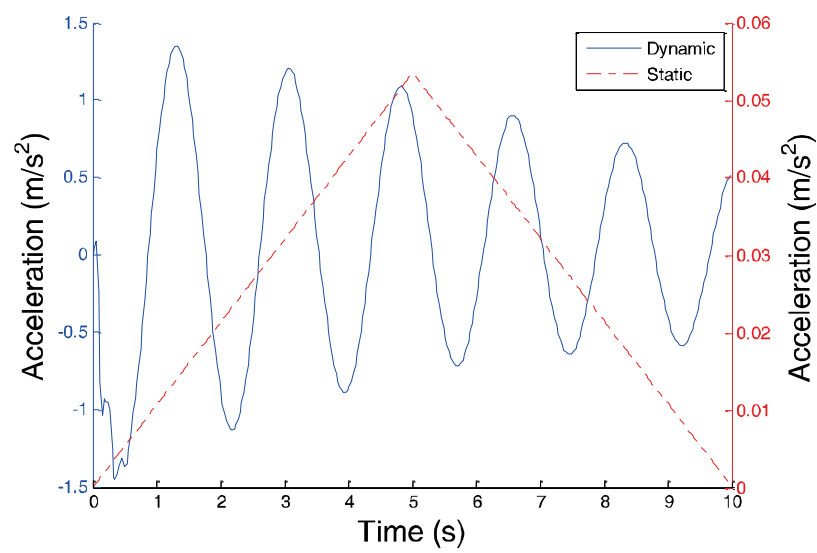

(b)

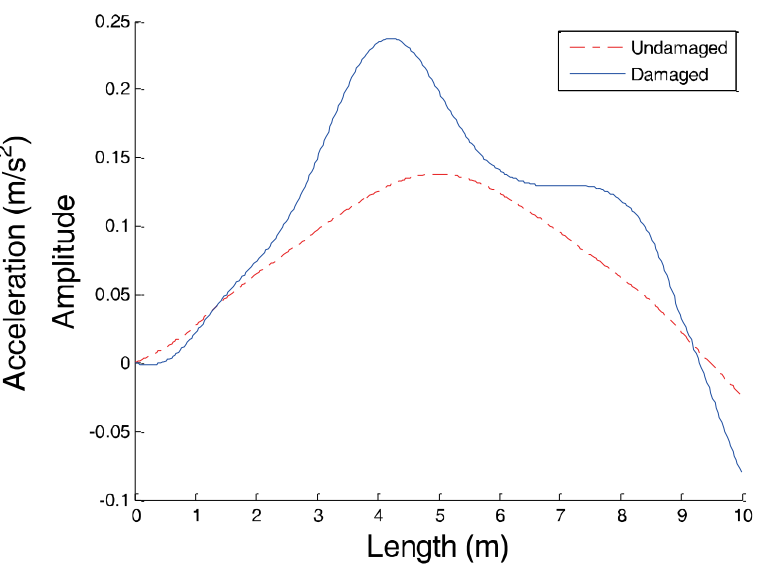

(d)

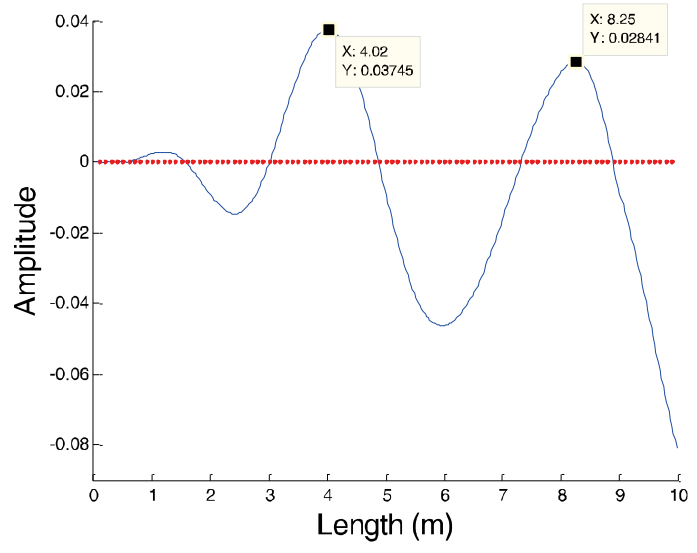

(e)

Fig. 4. (a) total mid-span acceleration of a healthy beam due to a load travelling at $30 \mathrm{~m} / \mathrm{s}$; (b) static and dynamic components of acceleration from Fig. 4a; (c) static and dynamic components of acceleration from Fig. 3c; (d) total healthy and total damaged accelerations after MAF is applied; (e) IMF1 of damaged acceleration from Fig. 4d.

component. The static component refers to the displacement, twice differentiated with respect to time, that would be experienced at mid-span if the load was statically applied and moved incrementally across the structure according to 
a given load velocity. The dynamic component is the displacement, twice differentiated with respect to time, due to the inertial forces of the beam. In the case of the acceleration of the healthy beam, the theoretical static component of acceleration would have a triangular shape given the parabolic shape of the theoretical static deflection (Fig. 4b). If the beam had experienced a loss of stiffness in a given location, both static and dynamic components of accelerations will be altered. In the case of the static component, there will appear a hump at the damaged zone in addition to the triangular shape associated to the healthy response (Fig. 4c). Here, the combination of both MAF (which removes most of the dynamic component as shown in Fig. 4d) followed by EMD (which removes most of the healthy static component while leaving a significant contribution associated to damage as shown in Fig. 4e) enhance the hump due to the damage while hindering dynamic and healthy static components. It must be noted that the MAF of the healthy acceleration response in Fig. $4 \mathrm{~d}$ is a monocomponent signal, i.e., it cannot be decomposed in further IMFs.

To implement the approach, first a FFT of the acceleration signal is used to establish the dominant frequency of the beam, and the span of the MAF is set equal to the number of scans associated to this dominant period of vibration (For Figs $4 \mathrm{a}$ - healthy beam- and 3c - damaged beam- these frequencies correspond to $17.96 \mathrm{~Hz}$ and $13.97 \mathrm{~Hz}$ respectively). Before applying the MAF the signal is padded at either end. The MAF is then applied twice to ensure full removal of the dominant frequency of the signal while smoothing higher frequencies associated to modes of vibration of the structure, the static component and any discontinuities [16]. EMD is then employed to create a monocomponent signal which has zero mean. The first IMF of the averaged signal (Fig. 4e) gives clearer damage peak locations than the averaged signal itself (Fig. 4d) for two reasons. Firstly, the underlying healthy triangular shaped static component in the acceleration signal is defined mostly within a low frequency band that is removed in the first IMF. Secondly, according to Huang et al. [6], when finding an IMF a gentle hump on a slope can be amplified to become a local extremum in changing the local zero from a rectangular to a curvilinear coordinate system. After the first round of sifting, the hump may become a local maximum. The peaks obtained above the zero mean line in the IMF representation of the filtered signal (Fig. 4e) appear to be clearer, more accurate and easier to interpret than the results of the traditional approach in Section 3.1 (Fig. 3b).

Figure 5a shows the acceleration signals, of a beam, after a MAF has been applied, with load velocities of 10, 20 and $30 \mathrm{~m} / \mathrm{s}$ over a $10 \mathrm{~m}$ beam span. As in the preceding simulation, the beam has two cracks of $\delta=0.3$ at $4 \mathrm{~m}$ and $8 \mathrm{~m}$ from the first support. The first IMF of the signals in Fig. 5a is shown in Fig. 5b. Only the parts of the IMFs above the zero mean line are represented for clarity of results. As expected, higher load velocities lead to higher peaks in the IMFs. Althozugh there are small peaks occurring at the start of the signal due to the start-up discontinuity as the load moves onto the bridge, the peaks denoting the presence of damage are visualized easily.

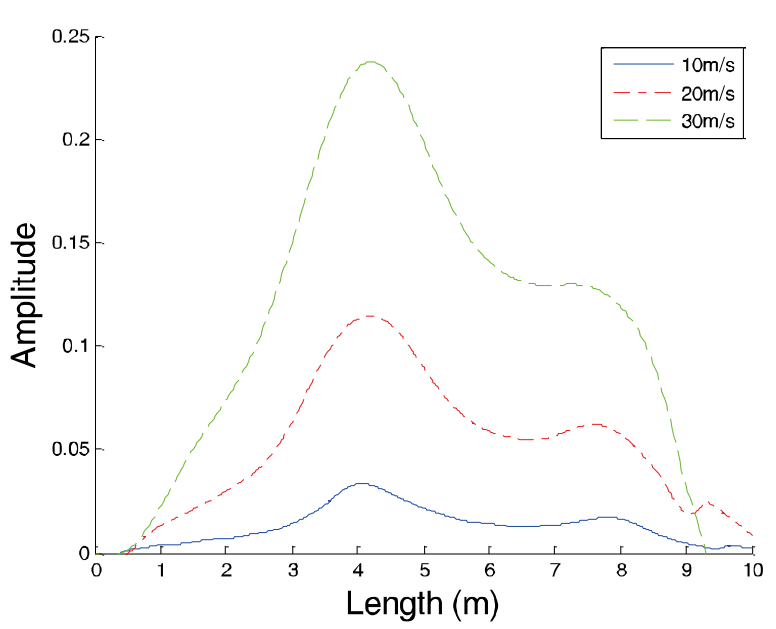

(a)

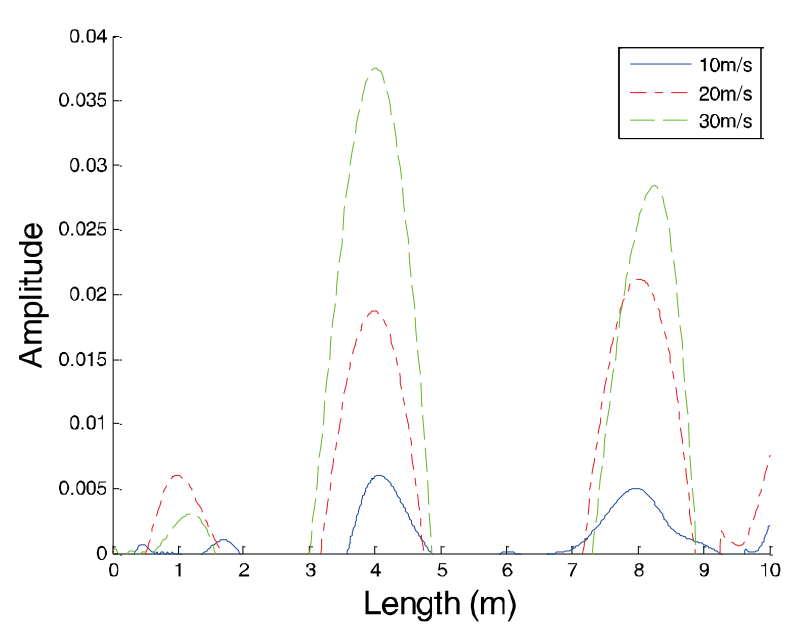

(b)

Fig. 5. (a) Acceleration signals with two cracks at 4 and $8 \mathrm{~m}$ after MAF is applied, (b) IMF 1 of signals in Fig. 5a. 
A range of parameters are examined next to give an indication of how this new algorithm will perform under a variety of scenarios. Beam models are tested with $\delta$ values of $0.3,0.2$ and 0.1 under varying load velocities, beam lengths, noise levels and damage locations. Finally the effect of the size of the area of influence of the crack and the positioning of the sensor on the results are tested.

\section{Results}

\subsection{Varying damage severity}

The proposed approach has shown to be capable of detecting two simultaneous damages at load velocities of 10 , 20 and $30 \mathrm{~m} / \mathrm{s}$ for $\delta=0.3$ (Fig. 5). Similar results are obtained at 10 and $20 \mathrm{~m} / \mathrm{s}$ with $\delta=0.2$ (Fig. 6a) and 0.1 (Fig. 6b). It is found that the lower the speed the sharper the damage peak is. However, detecting locations with these low levels of damage is not as accurate at the higher velocity of $30 \mathrm{~m} / \mathrm{s}$ where a loss in detail occurs. In the latter, it is difficult to separate frequency content due to damage from dynamic and healthy static components due to the reduced period of measurements.

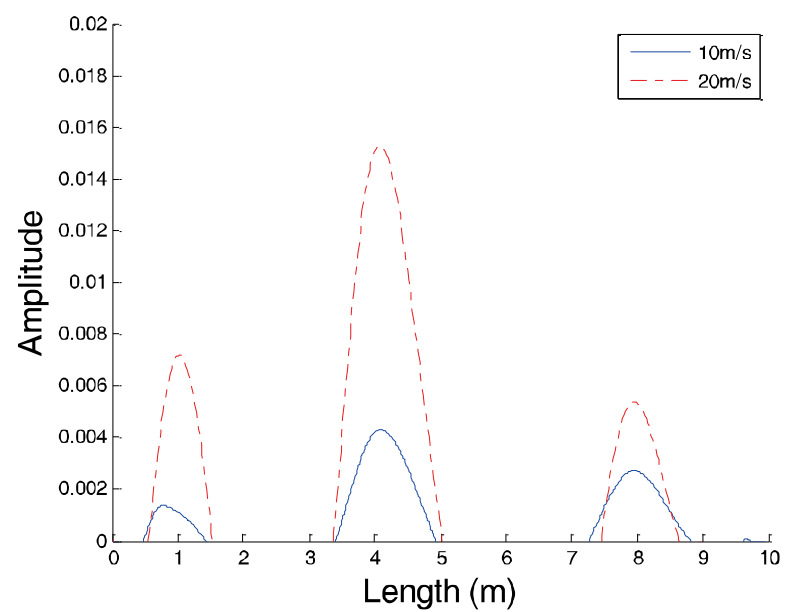

(a)

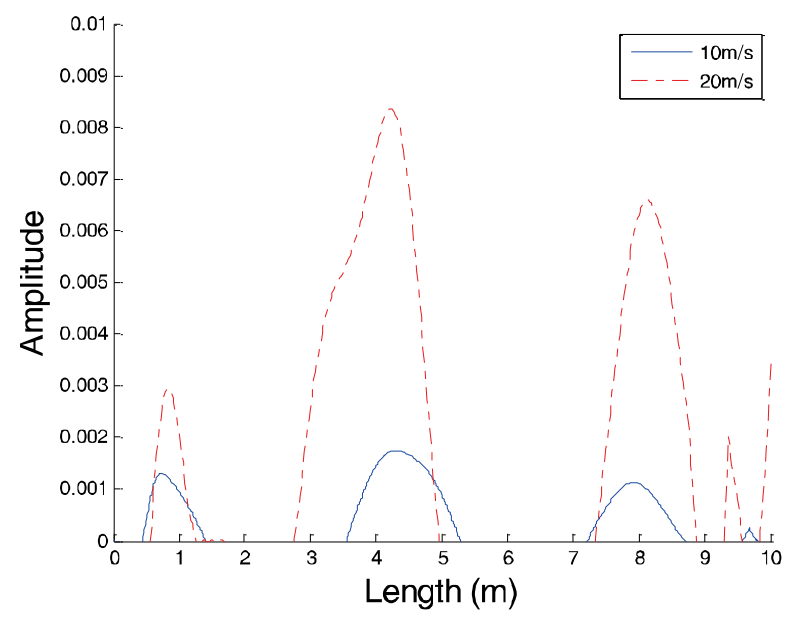

(b)

Fig. 6. IMF 1 from load velocities of 10 and $20 \mathrm{~m} / \mathrm{s}$ with $\delta$ of (a) 0.2 and (b) 0.1 at $4 \mathrm{~m}$ and $8 \mathrm{~m}$.

\subsection{Varying beam length}

Beam lengths of 20 and $30 \mathrm{~m}$ are tested in this section to determine if the variation in length has any effect on the algorithm's ability to detect damage. The load is set at a velocity of $10 \mathrm{~m} / \mathrm{s}$. Figures $7 \mathrm{a}$ and $7 \mathrm{~b}$ show the algorithm is capable of detecting damage in 10 and $20 \mathrm{~m}$ long beams. Figure $7 \mathrm{c}$, however, shows that although the damage peaks can be located in a $30 \mathrm{~m}$ beam, non-damage related peaks of smaller magnitude may develop. These non-damage related peaks can be removed by employing more than one sensor location when only the true damage peaks will appear in all sensors.

\subsection{Varying noise levels}

To take into consideration more realistic measurement conditions, the results of a $10 \mathrm{~m}$ beam with a $\delta$ of 0.3 traversed by a load at $10 \mathrm{~m} / \mathrm{s}$ are corrupted with white noise. Signal to Noise Ratios (SNRs) of 10 and 5 (i.e., relative errors in the measurements of $10 \%$ and $20 \%$ respectively) are examined in Figs $8 \mathrm{a}$ and $8 \mathrm{~b}$ respectively. Figure $8 \mathrm{~b}$ 


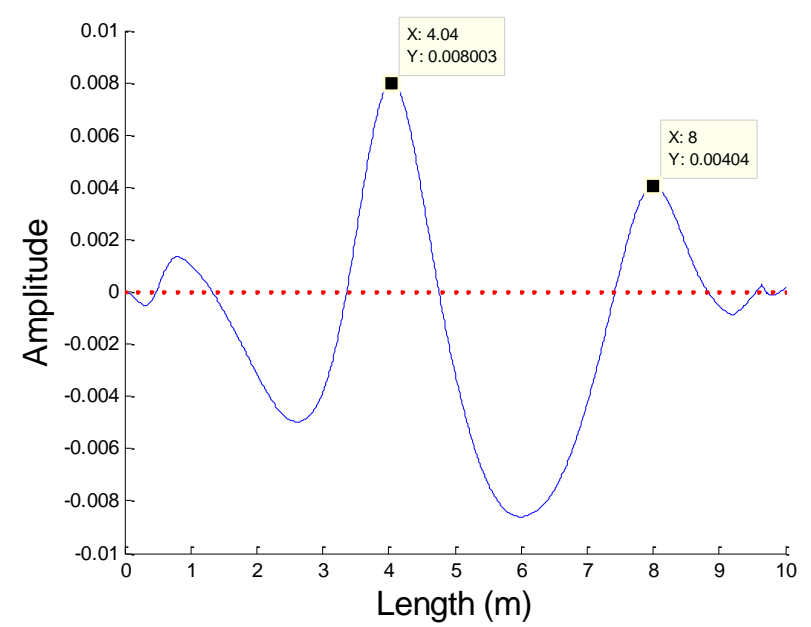

(a)

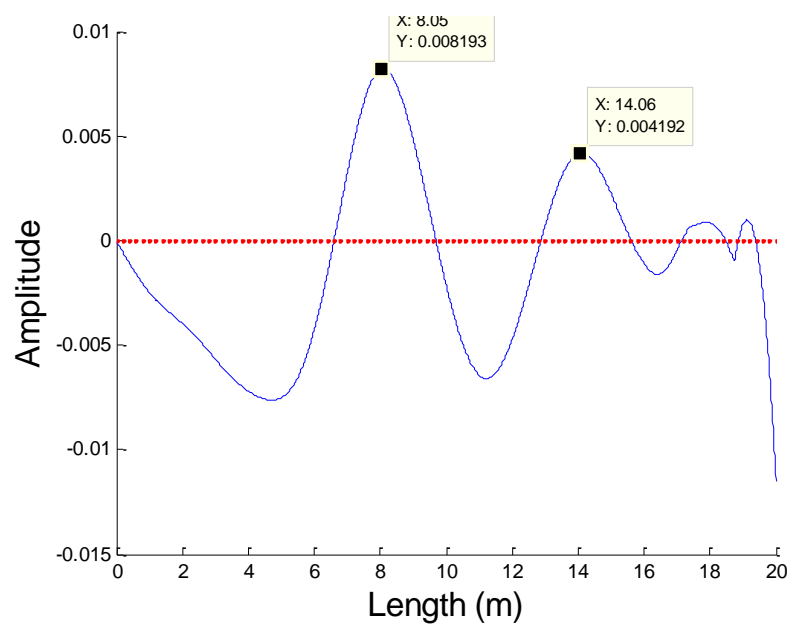

(b)

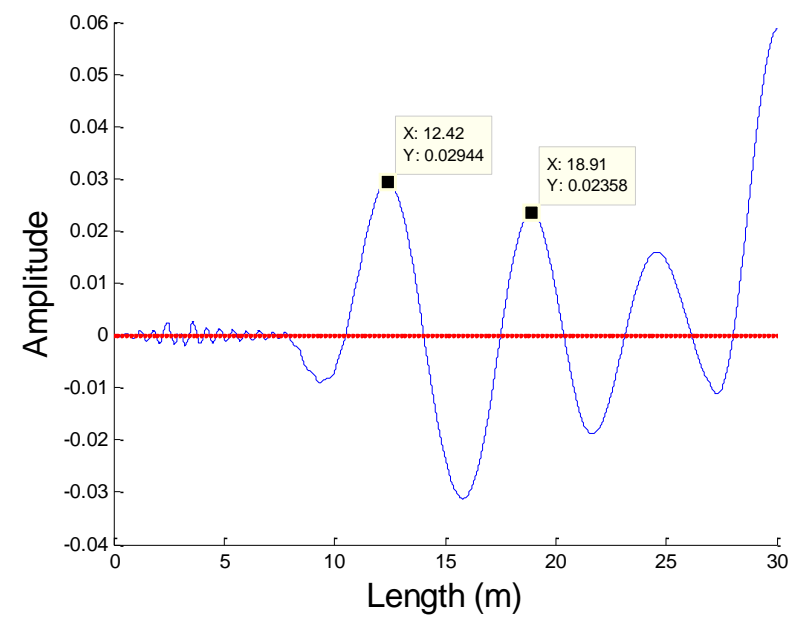

(c)

Fig. 7. IMF 1: (a) $\delta=0.3$ at $4 \mathrm{~m}$ and $8 \mathrm{~m}$ for a beam length of $10 \mathrm{~m}$, (b) $\delta=0.3$ at $8 \mathrm{~m}$ and $14 \mathrm{~m}$ for a beam length of $20 \mathrm{~m}$, (c) $\delta=0.3$ at $12 \mathrm{~m}$ and $19 \mathrm{~m}$ for a beam length of $30 \mathrm{~m}$.

shows that the accuracy of damage location for a SNR of 5 is redsuced due to deviations introduced by noise, but it can still be located within $0.35 \mathrm{~m}$.

\subsection{Varying damage proximity}

The position of the cracks is varied to determine the effect of their proximity on the performance of the algorithm. There is only a slight loss of accuracy for those cracks farthest apart from the measurement location, which is explained in more detail in Section 4.6. Figure 9a shows two clear peaks very close to the true crack locations at 1.5 and $8 \mathrm{~m}$. It is found that cracks in excess of $1 \mathrm{~m}$ from each other are distinguishable from one another, however within closer proximity the peaks will merge. This is due to the fact that the cracks themselves now merge as a result of the crack modelling method employed. An example where the cracks are at $6.5 \mathrm{~m}$ and $8 \mathrm{~m}$ along a $10 \mathrm{~m}$ beam is shown in Fig. 9b, again the damage is clearly located with sharp peaks. 


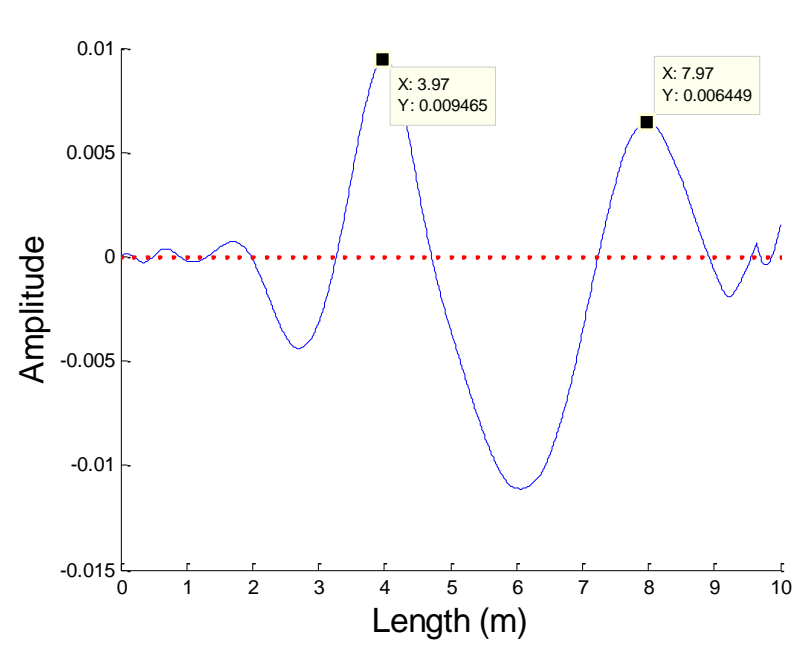

(a)

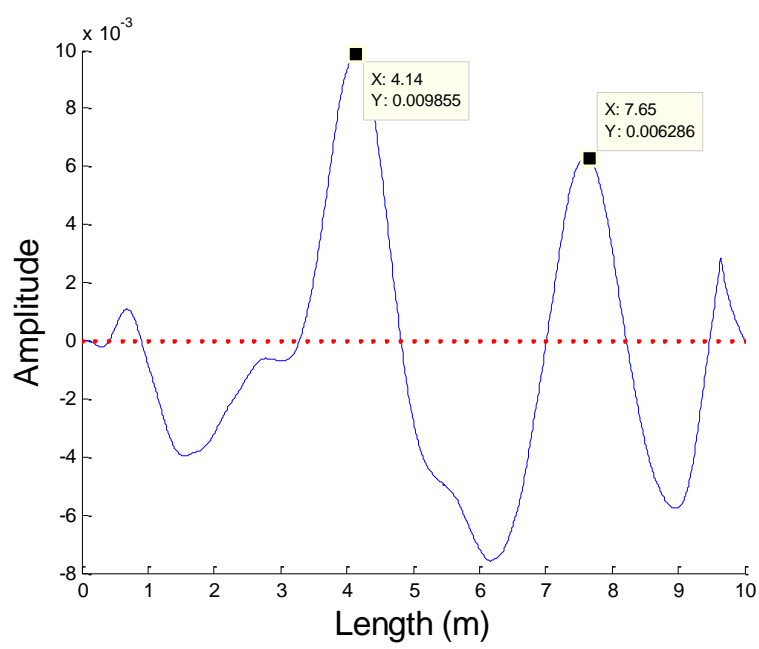

(b)

Fig. 8. IMF 1 with $\delta=0.3$ at $4 \mathrm{~m}$ and $8 \mathrm{~m}$ and (a) $\mathrm{SNR}=10$ and (b) $\mathrm{SNR}=5$.

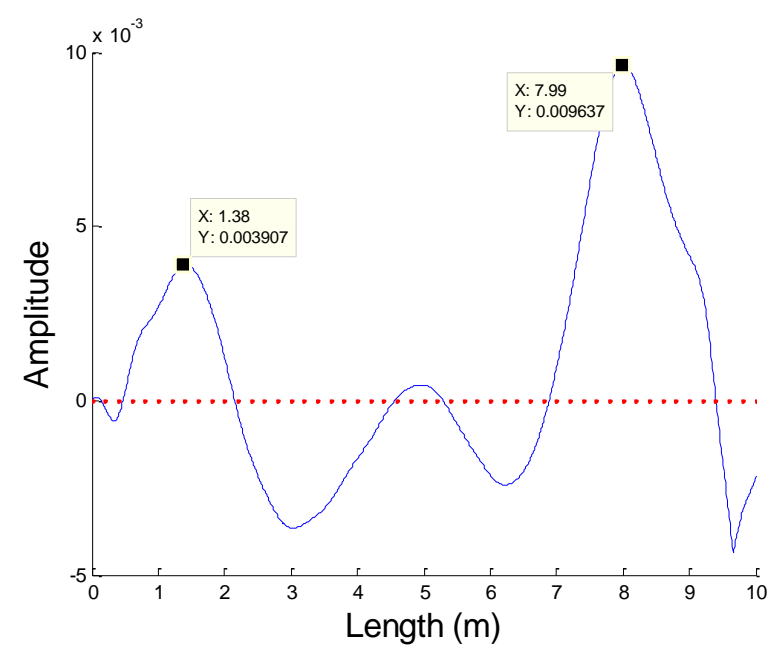

(a)

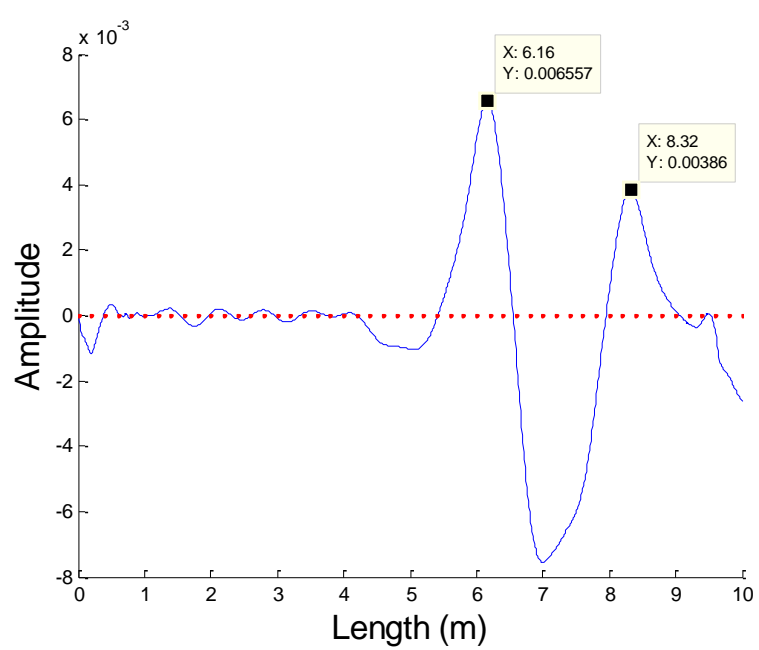

(b)

Fig. 9. IMF 1 with $\delta=0.3$ at (a) $1.5 \mathrm{~m}$ and $8 \mathrm{~m}$ and (b) $6.5 \mathrm{~m}$ and $8 \mathrm{~m}$

\subsection{Effect of area of influence of crack}

The model adopted for the crack in this study covers a triangular area of length $3 d$ (Fig. 2) which it is a relatively large proportion of the total beam length, particularly for the $10 \mathrm{~m}$ span. Another approach to modelling crack damage is to divide the beam into two parts that are pinned at the crack location and the crack is simulated by the addition of a rotational spring. Sinha et al. [15], however state that this is a gross simplification of the crack dynamics and does not involve the crack size and location directly. Therefore, one third of the length of influence of the crack previously used is adopted in this section. The results shown in Fig. 10 are for the same model set-up as shown in Figs $7 \mathrm{a}$ and $7 \mathrm{~b}$, where the velocity is $10 \mathrm{~m} / \mathrm{s}$ and damage is modelled with a $\delta$ of 0.3 , but this time, the area of influence of the crack spreads only $0.5 d$ either side of the crack location. It can be seen that even with small areas of influence from cracks, the algorithm is still capable of detecting the damage. 


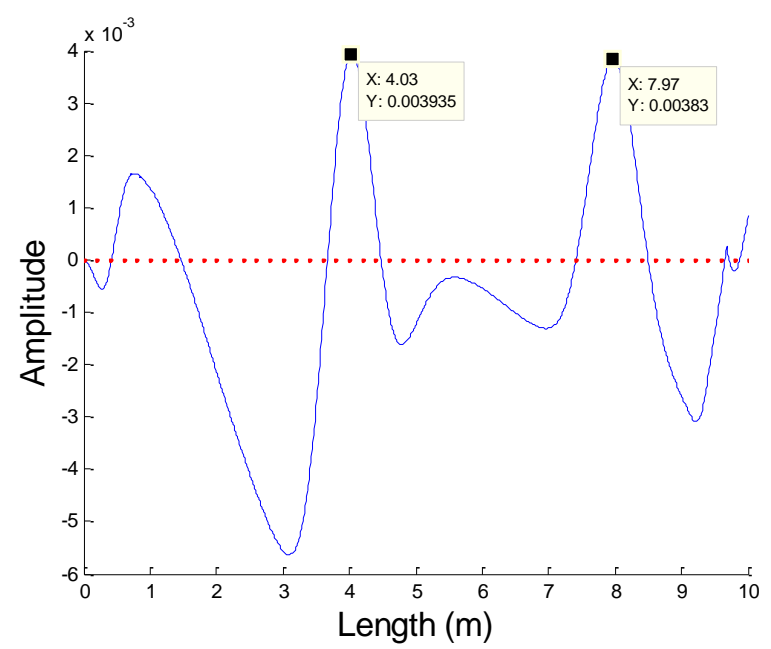

(a)

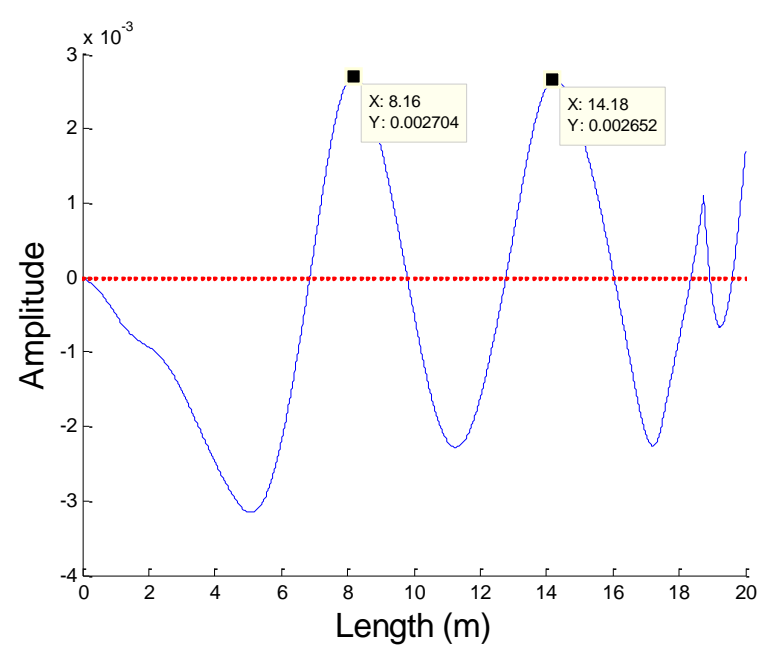

(b)

Fig. 10. IMF 1: (a) $\delta=0.3$ at $4 \mathrm{~m}$ and $8 \mathrm{~m}$ for beam length of $10 \mathrm{~m}$, (b) $\delta=0.3$ at $8 \mathrm{~m}$ and $14 \mathrm{~m}$ for beam length of $20 \mathrm{~m}$.

\subsection{Influence of sensor position}

Figure 11 illustrates the effect of sensor position on the height of the damage peak. The results in this figure have been obtained from processing accelerations at different measurement locations for a load travelling at $10 \mathrm{~m} / \mathrm{s}$ on a $20 \mathrm{~m}$ beam with a crack at the mid-span section. As the sensor moves away from the damage, from 0 (at the crack) to $10 \mathrm{~m}$ (at the support), the peak height diminishes. Damage can be detected across most of the beam length, however the damage peak heights merge for lower damage values beyond a distance of $4 \mathrm{~m}$, meaning the level of damage becomes unclear. If a number of measurement locations were available, the algorithm would be able to reduce uncertainties introduced by noise or a relative insensitive measurement location which is just too far apart from the crack.

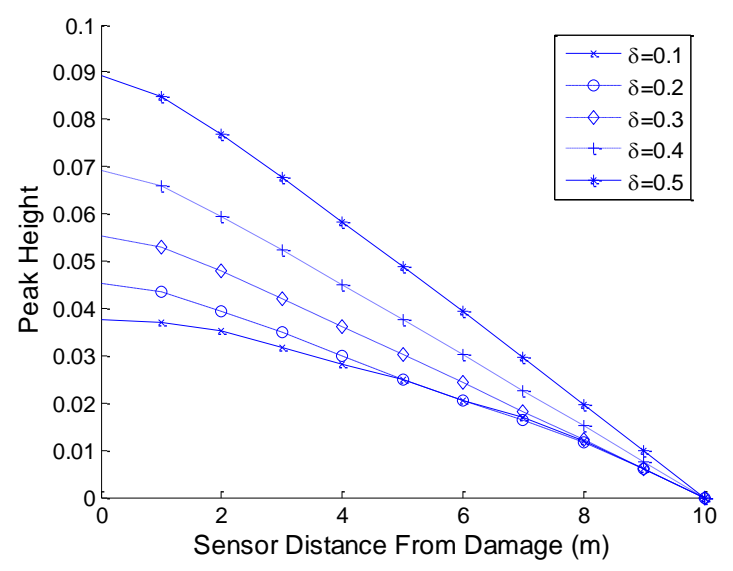

Fig. 11. Maximum damage peak height versus distance of sensor from damage location.

\section{Conclusion}

This paper has investigated the possibility of applying EMD to the acceleration response of a beam subject to a moving load to detect damage. It has been found, that on its own, EMD is capable of detecting multiple cracks, 
however, the results are unclear and disrupted by filter transients. A new approach has been employed that first applies a MAF to the signal before performing EMD. It is a signal-based model-free and adaptive method requiring no prior knowledge of the structure, which utilizes not only the nonlinear characteristic of the response to determine the damage, but also the transient properties of the load to determine the damage location. This method has been shown to be capable of detecting a crack of $10 \%$ of the beam depth in a signal generated at a velocity of $10 \mathrm{~m} / \mathrm{s}$ and corrupted with a SNR of 5. Therefore, the performance of the method can be improved through the use of multiple sensors which will facilitate to distinguish damage and non-damage related discontinuities and improve the accuracy of the predicted damage.

\section{Acknowledgments}

This research is supported by a scholarship from the Irish Research Council for Science, Engineering and Technology (IRCSET).

\section{References}

[1] L.W. Salvino, D.J. Pines, M. Todd and J.M. Nichols, EMD and instantaneous phase detection of structural damage, Hilbert-Huang Transform and Its Applications, Chapter 11, 2005, pp. 227-259.

[2] S. Doebling, C. Farrar and M. Prime, A summary review of vibration-based damage identification methods, The Shock and Vibration Digest 30(2) (1998), 91-105.

[3] H. Kim and H. Melhem, Damage Detection of Structures by Wavelet Analysis, Engineering Structures 26(3) (2004), $347-362$.

[4] X. Zhu and S. Law, Wavelet-based crack identification of bridge beam from operational deflection time history, International Journal of Solids and Structures 43(7-8) (2006), 2299-2317.

[5] A. Ayenu-Prah and N. Attoh-Okine, Comparative study of hilbert-huang transform, fourier transform and wavelet transform in pavement profile analysis, Vehicle System Dynamics 47(April 2009), 437-456.

[6] N.E. Huang, S. Shen, S. Long, M. Wu, H. Shih, Q. Zheng, N. Yen, C. Tung and H. Liu, The empirical mode decomposition and the Hilbert spectrum for nonlinear and non-stationary time series analysis, Proc R Soc London Ser A 454 (1998), 903-995.

[7] N.E. Huang and S.S.P. Shen, Hilbert-Huang transform and its applications, World Scientific Publishing, 2005.

[8] J.N. Yang, Y. Lei, S. Lin and N. Huang, Hilbert-huang based approach for structural damage detection, Journal of Engineering Mechanics 130(1) (2004), 85-95.

[9] X.L. Xu and J. Chen, Structural damage detection using empirical mode decomposition: Experimental investigation, Journal of Engineering Mechanics 130(11) (2004), 1279-1288.

[10] D. Pines and L. Salvino, Structural health monitoring using empirical mode decomposition and the hilbert phase, Journal of Sound and Vibration 294 (2006), 97-124.

[11] D. Yinfeng, L. Yingmin and L. Ming, Structural damage detection using empirical mode decomposition and vector autoregressive moving average model, Soil Dynamics and Earthquake Engineering 30 (2010), 133-145.

[12] M. Bradley, A. González and D. Hester, Analysis of The Structural Response of a Moving Load using Empirical Mode Decomposition, Proceedings of the $5^{\text {th }}$ International Conference on Bridge Maintenance, Safety, Management and Life-Cycle Optimization, IABMAS2010, Philadelphia, July 2010, pp. 356-363.

[13] N. Roveri and A. Carcaterra, Damage detection in structures under travelling loads by hilbert-huang transform, Mechanical Systems and Signal Processing 28 (2012), 128-144.

[14] J.W. Tedesco, W.G. McDougal and C. Allen Ross, Structural dynamics: theory and applications, Addison Wesley Longman, 1999.

[15] J.K. Sinha, M.I. Friswell and S. Edwards, Simplified models for the location of cracks in beam structures using measured vibration data, Journal of Sound and Vibration 251(1) (2002), 13-38.

[16] D. Hester and A. González, Detecting Damage in a Beam Subject to a Moving Load using Localised Variation in Vibration Response, Bridge and Infrastructure Research in Ireland, Cork, September 2010, pp. 307-314. 

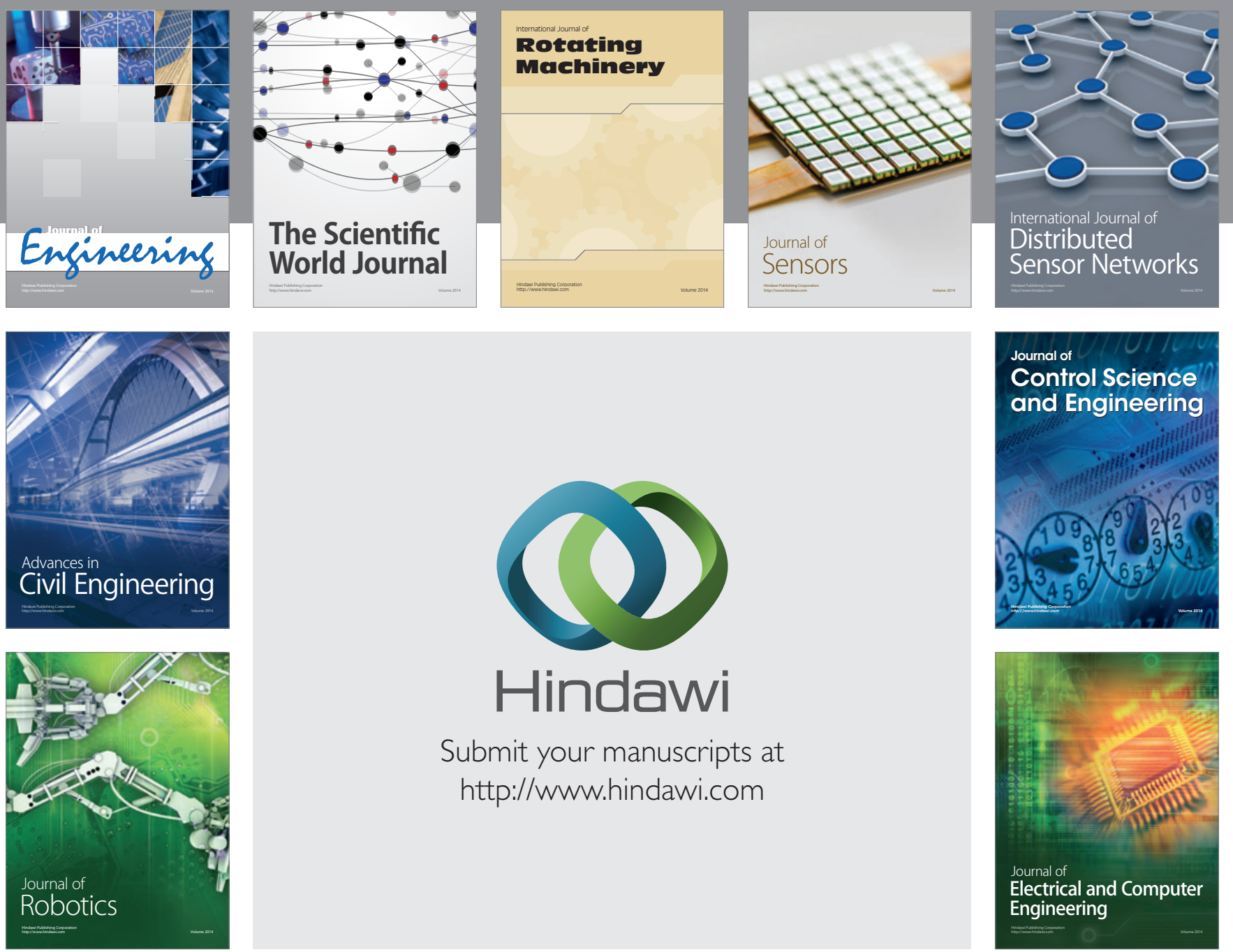

Submit your manuscripts at

http://www.hindawi.com
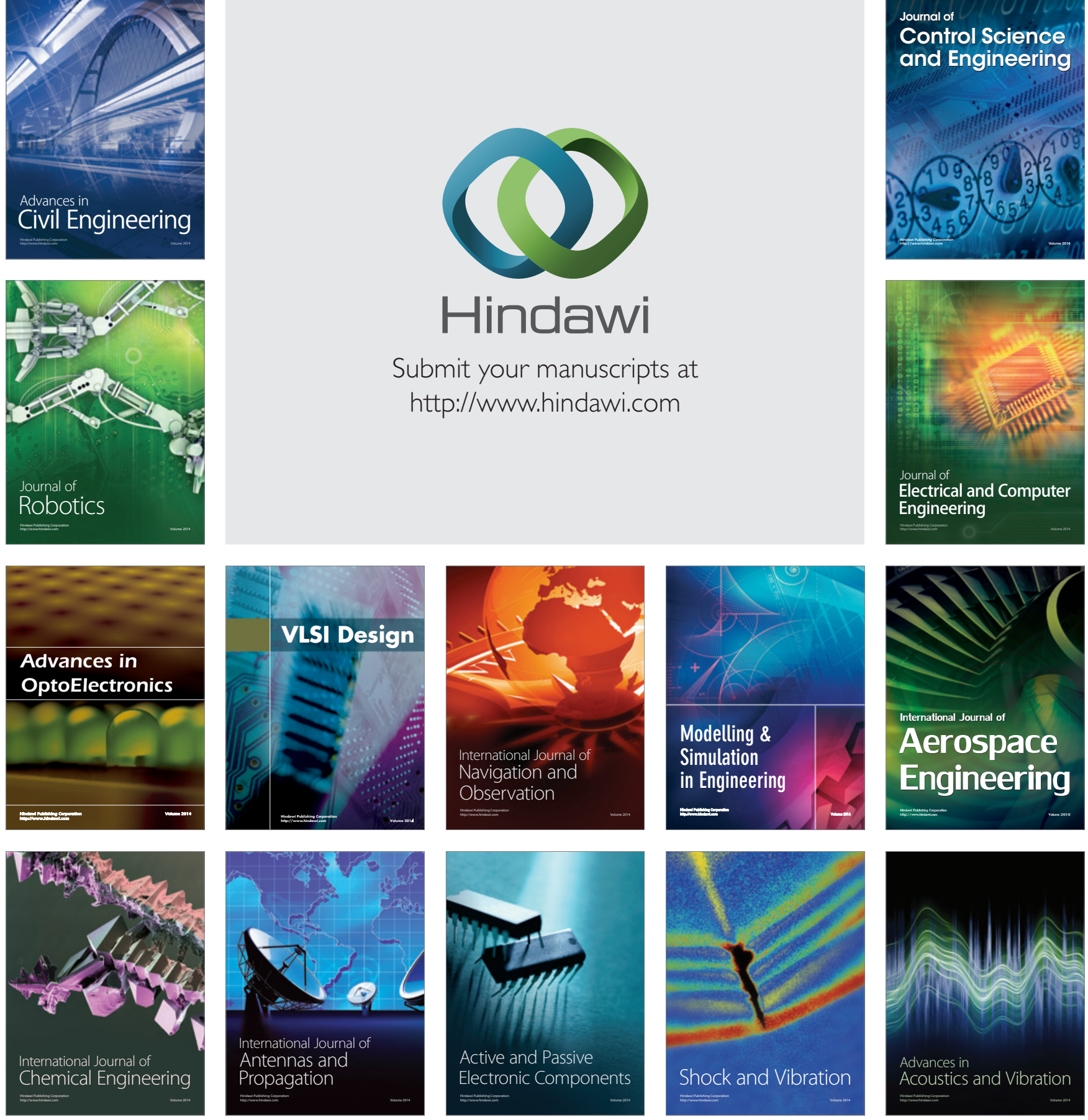